\title{
Comments on: Extensive facility location problems on networks: an updated review
}

\author{
Juan A. Mesa ${ }^{1}$
}

Published online: 9 April 2018

(C) Sociedad de Estadística e Investigación Operativa 2018

First of all, when reading the paper I have found impressive and interesting information about the location of extensive facilities in graphs, which shows the deep knowledge of the authors about the topic. When Brian Boffey and I decided to revise the literature on the location of facilities that are too large regarding their environment to be represented as isolated points, almost 25 years ago, we could not imagine the important development this line of research would reach nowadays. However, we had the feeling that reviewing this topic would open new avenues for the research in location analysis. Some of our suggestions have been studied, but others still remain to be researched as mentioned in the new review, such as for example some multiple path location problems on trees, and other problems which have arisen.

I agree with the limitations imposed on the problems revised in this paper. They do not deal with the location of extensive facilities in continuous spaces, since the nature of the underlying space leads to approaches coming from different mathematical areas, neither do they consider the location of repulsive and obnoxious extensive facilities because the basis for the techniques for solving these problems depends on the modelling of the involved application. The same reason applies to the location of cycles in graphs for which techniques based on mixed integer mathematical programming are usual, and finally the paper is dedicated to exact and approximations algorithms but not to heuristics. With these limitations, the aim of the paper is to provide a review of those works that explore the structure of the underlying graph along with the properties

This comment refers to the invited paper available at https://doi.org/10.1007/s11750-018-0476-5.

$\triangle \quad$ Juan A. Mesa

jmesa@us.es

1 Departamento de Matemática Aplicada II, Escuela Técnica Superior de Ingeniería, Universidad de Sevilla, Camino de los Descubrimientos, s/n, 41092 Seville, Spain 
of the objective function. In this sense, the framework of the research included in this review has been clearly defined and the focus of attention well stated.

The paper has two differentiated parts: the first one is dedicated to revising the general techniques applied to these problems, and the second is a survey of the work already done both for the location of paths and subtrees. They conclude with some conclusions where they highlight that subtree location on graphs is definitely less developed than that of paths.

Regarding the objective functions, the authors revise papers dealing with the eccentricity (center), distsum (median), combination of the eccentricity and distsum functions, equity measures, minimum loss, and ordered median. In point location, there is another kind of problem: those involving the coverage of demand points. In these problems, a demand point is (indirectly) covered if the distance to the facility is less than or equal to a given value called the covering radius. When the coverage function, the number of covered (weighted) points, is combined with some measure of the size of the facility (typically the number of facilities to be opened in point location theory), two problems result depending on which is the objective and which is the constraint. When the objective is to minimize the size of the facility subject to that all the demand points would be covered, the problem is known as the covering problem. When the objective is to maximize the coverage function constrained to a bound on the size of the facility, the problem is known as the coverage problem. Coverage is commonly used in transportation network design as a surrogate of the future utilization (known as the ridership) of the network. The research on extensive facility covering problems is however scarce. As far as I am aware, the first paper dealing with covering by extensive facilities in graphs is Kincaid et al. (1988), where the problems of finding a subtree of a tree minimizing the number of vertices or the length of the facility subject to all the vertices of the tree to be covered were dealt with in it. Subsequently, these problems were studied in Kim et al. (1989, 1990, 1996). More recently, an optimal $\mathrm{O}(n \log n)$ time algorithm has been proposed in Spoerhase (2010) for the indirect covering subtree problem. Continuing with potential applications to network design problems, when the demand is given by pairs of vertices (trips to be done for example), the location of extensive facilities where the speed is higher than in the rest of the network is important. For this problem, in the paper by Schmidt and Schöbel (2014), the computational complexity of several cases of the location of a subnetwork, so that the maximum time of the OD pairs is minimized, is obtained.

\section{References}

Kincaid R, Lowe T, Morin T (1988) The location of central structures in trees. Comput Oper Res 15(2):103111

Kim T, Lowe T, Ward J, Francis R (1989) A minimum length covering subgraph of a network. Ann Oper Res 18:245-260

Kim T, Lowe T, Ward J, Francis R (1990) A minimum-length covering subtree of a tree. Nav Res Logist 37:309-326

Kim T, Lowe T, Tamir A, Ward J (1996) On the location of a tree-shaped facility. Networks 28:167-175

Spoerhase J (2010) An optimal algorithm for the indirect covering subtree problem. arXiv:1002.0580v1

Schmidt M, Schöbel A (2014) Location of speed-up subnetworks. Ann Oper Res 223(1):379-401 